\title{
ROCK-phosphorylated vimentin modifies mutant huntingtin aggregation via sequestration of IRBIT
}

Peter O Bauer ${ }^{1,4+}$, Roman Hudec ${ }^{2,5+}$, Anand Goswami ${ }^{1}$, Masaru Kurosawa', Gen Matsumoto', Katsuhiko Mikoshiba ${ }^{2,3^{*}}$ and Nobuyuki Nukina ${ }^{1 *}$

\begin{abstract}
Background: Huntington's Disease (HD) is a fatal hereditary neurodegenerative disease caused by the accumulation of mutant huntingtin protein $(\mathrm{Htt})$ containing an expanded polyglutamine (polyQ) tract. Activation of the channel responsible for the inositol-induced $\mathrm{Ca}^{2+}$ release from ensoplasmic reticulum (ER), was found to contribute substantially to neurodegeneration in HD. Importantly, chemical and genetic inhibition of inositol 1,4,5-trisphosphate (IP3) receptor type 1 (IP3R1) has been shown to reduce mutant Htt aggregation.
\end{abstract}

Results: In this study, we propose a novel regulatory mechanism of IP3R1 activity by type III intermediate filament vimentin which sequesters the negative regulator of IP3R1, IRBIT, into perinuclear inclusions, and reduces its interaction with IP3R1 resulting in promotion of mutant Htt aggregation. Proteasome inhibitor MG132, which causes polyQ proteins accumulation and aggregation, enhanced the sequestration of IRBIT. Furthermore we found that IRBIT sequestration can be prevented by a rho kinase inhibitor, Y-27632.

Conclusions: Our results suggest that vimentin represents a novel and additional target for the therapy of polyQ diseases.

Keywords: Vimentin, IP3R1, IRBIT, Rho-kinase, Huntingtin, Aggregation

\section{Background}

Huntington's disease (HD) is an autosomal-dominant neurodegenerative disorder caused by CAG repeat expansion coding for a polyglutamine (polyQ) sequence in the $\mathrm{N}$-terminal region of the huntingtin protein $(\mathrm{Htt})$. The expansion of more than 36 repeats causes misfolding of the gene product huntingtin resulting in a toxic gain-of-function [1]. Clinically, HD is characterized by chronic and progressive involuntary choreiform movements, mood disorders, cognitive impairment, and behavioral changes [2,3]. A prominent feature of this disease is progressive neurodegeneration, with neuronal intranuclear and cytoplasmic accumulation of aggregated polyQ protein $[4,5]$. HD pathomechanism involves a broad scale of events including dysregulation of

\footnotetext{
* Correspondence: mikosiba@brain.riken.jp; nukina@brain.riken.jp

${ }^{\dagger}$ Equal contributors

${ }^{1}$ Laboratory for Structural Neuropathology, Brain Science Institute, RIKEN, 2-1

Hirosawa, Wako-shi, Saitama 351-0198, Japan

${ }^{2}$ Laboratory for Developmental Neurobiology, Brain Science Institute, RIKEN,

2-1 Hirosawa, Wako-shi, Saitama 351-0198, Japan

Full list of author information is available at the end of the article
}

transcription and gene expression, impairment of axonal transport and synaptic transmission and impairment of the ubiquitin proteasome system (UPS) [6,7]. Mitochondrial dysfunction leading to induction of mitochondrial apoptotic pathway has also been described in HD with $\mathrm{Ca}^{2+}$ mishandling and suppression of energy metabolism $[8,9]$. Despite an enormous effort in elucidating the pathogenesis of this disorder, effective therapies for HD have not yet been found.

Vimentin is a $57 \mathrm{kDa}$ type III intermediate filament (IF) found in cells of mesenchymal origin [10,11]. While widely expressed in embryos, vimentin is replaced by other major classes of IFs in cells during terminal differentiation $[12,13]$. In the adult brain, vimentin expression is mostly restricted to some subpopulations of glial and vascular endothelial cells under physiological conditions [12-14]. Importantly, it has been found that vimentin expression is re-activated in mature neurons affected by Alzheimer's disease or traumatic injury $[15,16]$.

Degradation of misfolded proteins has been shown partly mediated by UPS. The components of UPS including the $26 \mathrm{~S}$ proteasome and ubiquitin as well as heat

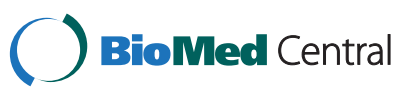


shock proteins are concentrated at the centrosome [17]. When UPS is overloaded by misfolded proteins and/or it is chemically inhibited, the centromeric accumulation of these proteins increases forming aggresomes which may represent a general cellular response to dysfunctional or damaged polyubiquitinated proteins accumulation $[18,19]$. Another evidence of the association of aggresome formation with the accumulation and degradation of misfolded proteins has come from studies, where pathogenic polyQ proteins Htt and atrophin-1 formed inclusions at centrosomes which were surrounded by vimentin [20,21]. Vimentin is recruited to the aggresomes during UPS dysfunction and forms a cage-like structure surrounding the pericentriolar focus of aggregated protein [19].

The role of aggresomes and especially the vimentin cage in polyQ diseases progression is not clear. We hypothesized that vimentin may play a major role in polyQ proteins accumulation and aggregation and that vimentin cage may immobilize or trap not only the UPS components and chaperones, but also other important proteins at the centrosomic inclusions and thus preventing their functions elsewhere in the cell. We found that one of such proteins, IP3R1-interacting protein released with IP3 (IRBIT) is also sequestered by vimentin. IRBIT has numerous regulatory functions among which the IP3R1 activity regulation is most intriguing [22,23]. IRBIT binds to IP3-binding core domain of IP3R1 acting as a competitor to IP3 $[23,24]$. Absence of IRBIT sensitizes IP3R1 to IP3, which leads to an increase in $\mathrm{Ca}^{2+}$ release from endoplasmic reticulum [23].

IP3R1 was found to be involved in polyQ diseases pathomechanism $[25,26]$. In planar lipid bilayer reconstitution experiments and in primary cultures of rat striatal medium spiny neurons, IP3R1 was sensitized to IP3 by mutant forms of $\mathrm{Htt}$, while normal $\mathrm{Htt}$ had no effect. This finding confirmed that the activation of IP3R1 by expanded polyQ $\mathrm{Htt}$ is a contributing factor of $\mathrm{Ca}^{2+}$ signaling alteration and neuronal degeneration in HD [25]. Knock-down of IP3R1 or direct chemical inhibition of the IP3R1 activity also reduced polyQ proteins accumulation and aggregation [27] and cell death [28].

Here we introduce a novel pathway of IP3R1 activity regulation, where vimentin is able to sequester IRBIT from interaction with IP3R1. Moreover, IRBIT sequestration was enhanced by the phosphomimetic S71E/S38E vimentin mutant (E2; Ser71 and Ser38 replaced with Glu). Phosphorylation of Ser71 and Ser38 is mediated by rho-associated kinases (ROCKs) [29,30]. ROCKs are Ser/ Thr protein kinases, which were found to be downstream targets of the small GTPase RhoA [31,32]. In the mammalian system, ROCKs consist of two isoforms, ROCK1 and ROCK2 [31]. They are important regulators of cell growth, migration, and apoptosis via control of actin cytoskeletal assembly [33]. Blocking the RhoA/
ROCK pathway has been shown to inhibit the polyQ protein aggregation and decrease its toxicity in cellular and Drosophila models of HD [34]. ROCK1 and protein kinase C-related protein kinase-2 (PRK-2) have been identified to be the mediators of aggregation reduction by the well-known ROCK inhibitor Y-27632 [35]. Moreover, a downstream effector of ROCK1, actin-binding factor profilin, was reported to inhibit the mutant Htt aggregation by direct interaction via its polyprolinebinding domain [36]. Previously, we have reported that Y-27632 treatment also reduced aggregation of several other polyQ proteins without polyproline tracts, thus possibly affecting additional targets $[37,38]$. Here we show that vimentin may represent one of the mediators of ROCK inhibition-dependent reduction of pathogenic polyQ proteins aggregation via modulation of IP3R1 activity by IRBIT.

\section{Results and discussion}

\section{Effect of vimentin levels and phosphorylation on polyQ aggregation}

To investigate the role of vimentin in polyQ Htt processing, we considered several clues. Firstly, UPS impairment is thought to contribute to the severity of HD $[6,7]$. Vimentin creates cages around aggresomes, which are formed in response to accumulation of misfolded proteins and UPS dysfunction [19]. Secondly, vimentin is phosphorylated by several kinases, including ROCKs $[29,30]$. Thirdly, ROCK1 is activated by dopamine through dopamine D2 receptor (D2R)-specific pathway potentiating the glutamate excitotoxicity in $\mathrm{HD}[39,40]$ and the genetic and chemical inhibition of Rho/ROCK signaling pathway reversed dopamine/D2R-mediated cellular pathology [40]. Importantly, ROCK inhibitor Y-27632 reduced mutant Htt levels and aggregation both in vitro $[34,37]$ and in vivo and improved motor impairment in R6/2 HD mouse model [41].

We overexpressed RFP or RFP-vimentin in 16Q and 60Q and 150Q Neuro2a cells. We observed that vimentin accumulated at perinuclear regions and formed cage-like structures around tNHtt-60Q-EGFP and tNHtt150Q-EGFP inclusions in 60Q and 150Q Neuro2a cells while RFP exerted diffuse distribution in all cell lines (Figure 1A and Additional file 1: Figure S1). This confirmed the previously reported colocalization of vimentin with pathogenic polyQ protein inclusions $[17,18]$.

Next we asked whether vimentin could modulate mutant Htt aggregation. We found that over-expression of RFP-vimentin in 150Q Neuro2a cells dramatically increased the accumulation of insoluble Htt. Accumulation of the soluble form was also observed and could be the result of enhanced aggresomes formation leading to suppression of UPS activity under this condition. Vimentin knock-down, on the other hand reduced the mutant 


\section{A}
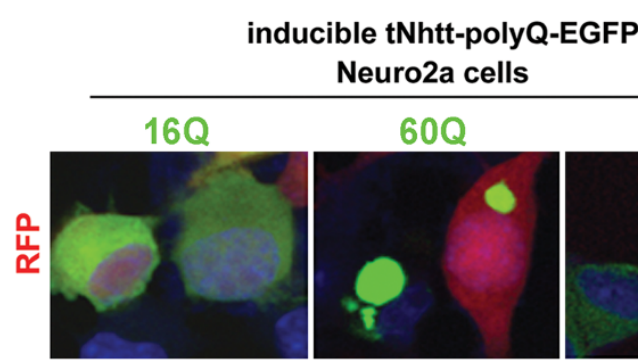

$60 Q$
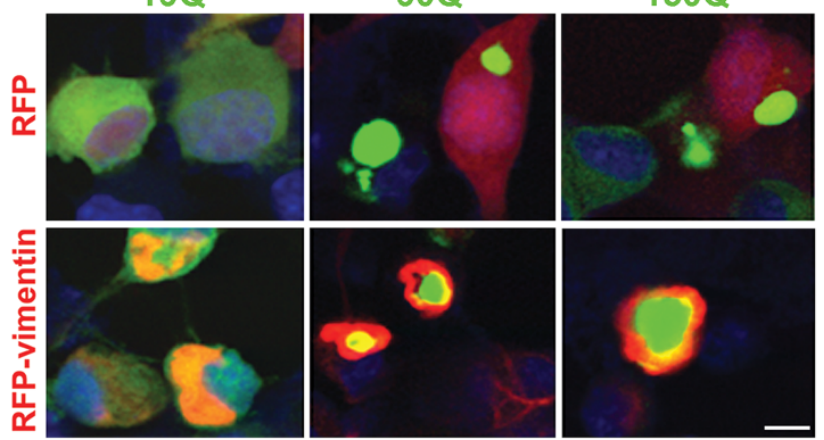

B

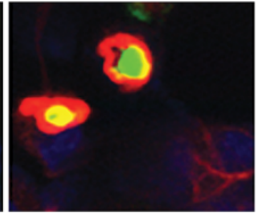

C

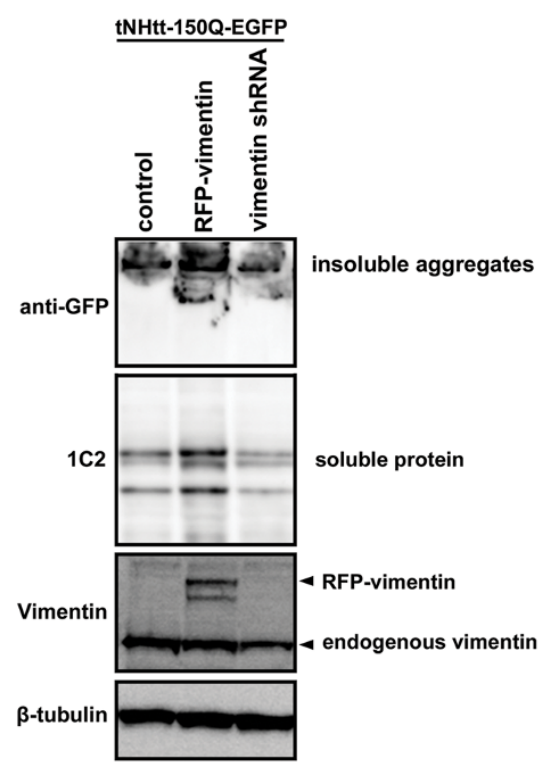

tNHtt-polyQ-EGFP $16 \mathrm{6} \quad 60 \mathrm{150Q}$

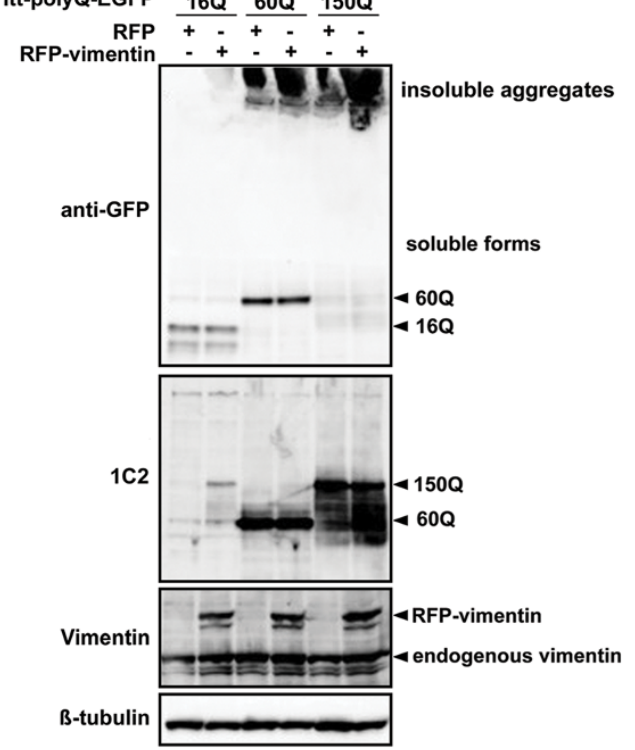

Figure 1 Vimentin modifies mutant $\mathrm{Htt}$ aggregation. A. Representative confocal images show distribution of normal (16Q) and pathogenic (60Q and 150Q) tNHtt (green) and RFP or RFP-vimentin (red) in inducible tNHtt-polyQ-EGFP Neuro2a cells. Note the cages formed by vimentin in $60 \mathrm{Q}$ and $150 \mathrm{Q}$ Neuro2a cells. Nuclei were stained with DAPI (blue). Scale bar, 5 mm. B. RFP-vimentin expression increased and vimentin knockdown reduced polyQ aggregation and levels of total mutant $\mathrm{Htt}$ in $150 \mathrm{Q}$ Neuro2a cells as compared to the control. C. The effect of RFP-vimentin on Htt levels is polyQ length-dependent. While tNHtt-60Q-EGFP and tNHtt-150Q-EGFP accumulated as the insoluble forms at the gel top, tNHtt16Q-EGFP levels remained unchanged upon RFP-vimentin transfection.

Htt aggregation (Figure 1B). To test whether the effect of vimentin is polyQ length-dependent, we overexpressed RFP-vimentin in 16Q, 60Q and 150Q Neuro2a cells. Vimentin appeared to act specifically on mutant $\mathrm{Htt}$, as the levels of tNHtt-16Q-EGFP remained unchanged while the accumulation of insoluble pool of the pathogenic Htt forms increased (Figure 1C).

Vimentin has been shown phosphorylated by ROCK at Ser71 and Ser38 amino residues [29,30] and we confirmed this fact, as treatment of Neuro2a cells with the ROCK inhibitor Y-27632 reduced the phosphorylation at these sites (Figure 2A). We transfected stable RFPvimentin Neuro2a cells with tNHtt-60Q-EGFP and treated them with Y-27632. Interestingly, we detected a modified subcellular distribution of stably expressed RFP-vimentin in Neuro2a cells treated with Y-27632 (Figure 2B). In the untreated cells, RFP-vimentin formed cage-like structures around tNHtt-60Q-EGFP inclusions while the Y-27632 treatment changed the localization of RFP-vimentin to neurites (Figure 2B). This observation suggested that vimentin phosphorylation by ROCK might influence polyQ aggregation. 

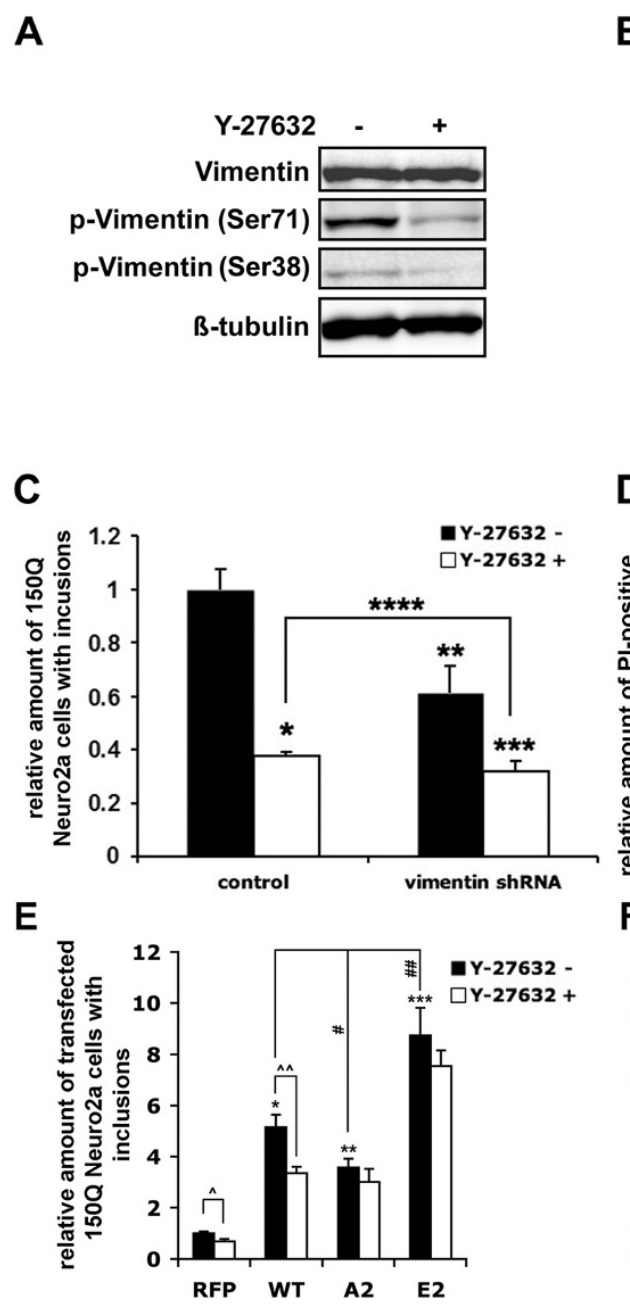

B

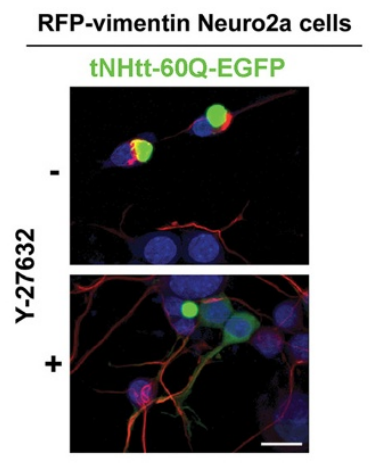

D

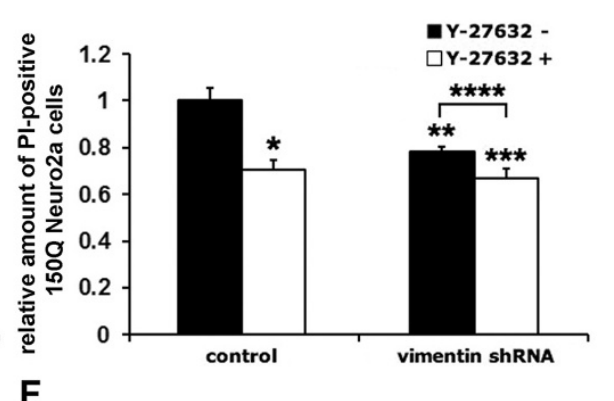

$\mathbf{F}$

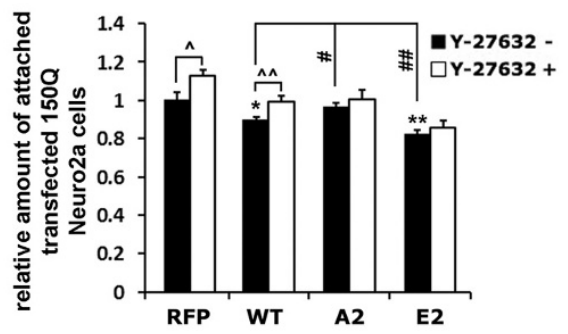

Figure 2 Vimentin affects the mutant $\mathrm{Htt}$ inclusion formation in $150 \mathrm{Q}$ Neuro2a cells and mediates the effect of Y-27632. A. Immunoblot demonstrating inhibition of vimentin phosphorylation at Ser71 and Ser38 by ROCK inhibitor Y-27632 (20 $\mu \mathrm{M})$ in Neuro2a cells. B. tNHtt-60Q-EGFP (green) was transfected to Neuro2a cells stably expressing RFP-vimentin (red). Treatment of these cells with $20 \mu \mathrm{M}$ Y-27632 resulted in filamentlike distribution of vimentin and disruption of vimentin cages observed around tNHtt-60Q-EGFP inclusions in the untreated cells. Nuclei were stained with DAPI (blue). Scale bar, $15 \mu \mathrm{m}$. C. The effect of Y-286432 on polyQ inclusion formation depends on vimentin level (high vimentin levels enhance inclusion formation). 150Q Neuro2a cells were transfected with vimentin shRNA and 48 hrs later, the cells were induced and treated with $20 \mu \mathrm{M}$ Y-27632. After 24 hrs, cells were fixed and the inclusion formation was quantified by ArrayScan. ${ }^{*} p=0.00003,{ }^{* *} p=0.0012$, ${ }^{* * *} p=0.00003,{ }^{* * *} p=0.0018$. D. The effect of vimentin knock-down and Y-27632 on polyQ cytotoxicity. Cells were incubated with PI and the PIpositivity was quantified by ArrayScan. ${ }^{*} p=0.0022,{ }^{* *} p=0.002,{ }^{* *} p=0.001,{ }^{* * *} p=0.017$. E. The effect of Y-286432 on polyQ inclusion formation is dependent on vimentin phosphorylation (vimentin phosphorylation enhances inclusion formation). 150Q Neuro2a cells were transfected with RFP, WT and phospho-mutant (A2 and E2) forms of RFP-vimentin. Cells were induced and treated with $20 \mu \mathrm{M} \mathrm{Y-27632} \mathrm{for} 24$ hrs, fixed and analyzed by ArrayScan. ${ }^{*} p=0.00016,{ }^{* *} p=0.0003,{ }^{* * *} p=0.0004,{ }^{\wedge} p=0.0021,{ }^{\wedge} p=0.018,{ }^{\#} p=0.04,{ }^{\# \#} p=0.022$. F. The effect of vimentin overexpression and Y-27632 on polyQ-induced cell death. Dead cells were detached and removed during samples preparation for analysis. Cells that remained attached were counted by ArrayScan. ${ }^{*} p=0.049,{ }^{* *} p=0.008,{ }^{\wedge} p=0.044,{ }^{\wedge \wedge} p=0.045,{ }^{\#} p=0.032,{ }^{\# \#} p=0.034$. Bars in C-F represent relative mean values \pm s.d. from three independent experiments, with levels under control conditions normalized to a value of 1.

To quantify the effects of vimentin levels on mutant Htt aggregation, we transfected the 150Q Neuro2a cells with vimentin shRNA and counted the inclusions on a cell-to-cell basis by ArrayScan. Vimentin knock-down reduced the number of the cells with inclusions by $39 \%$ (Figure 2C). Treatment of the 150Q Neuro2a cells with $20 \mu \mathrm{M}$ Y-27632 reduced the polyQ aggregation by $62 \%$, similarly to the previously reported effect in these cells
[37]. Vimentin knock-down significantly decreased the effect of Y-27632 to $40 \%$ (22\% difference as compared to the $62 \%$ aggregation reduction in the non-transfected cells) (Figure 2C), suggesting that the effect of Y-27632 is partly mediated through the inhibition of the phosphorylation of vimentin. Importantly, vimentin knock-down also significantly decreased the number of propidium iodide (PI)-positive 150Q Neuro2a cells indicating reduction of 


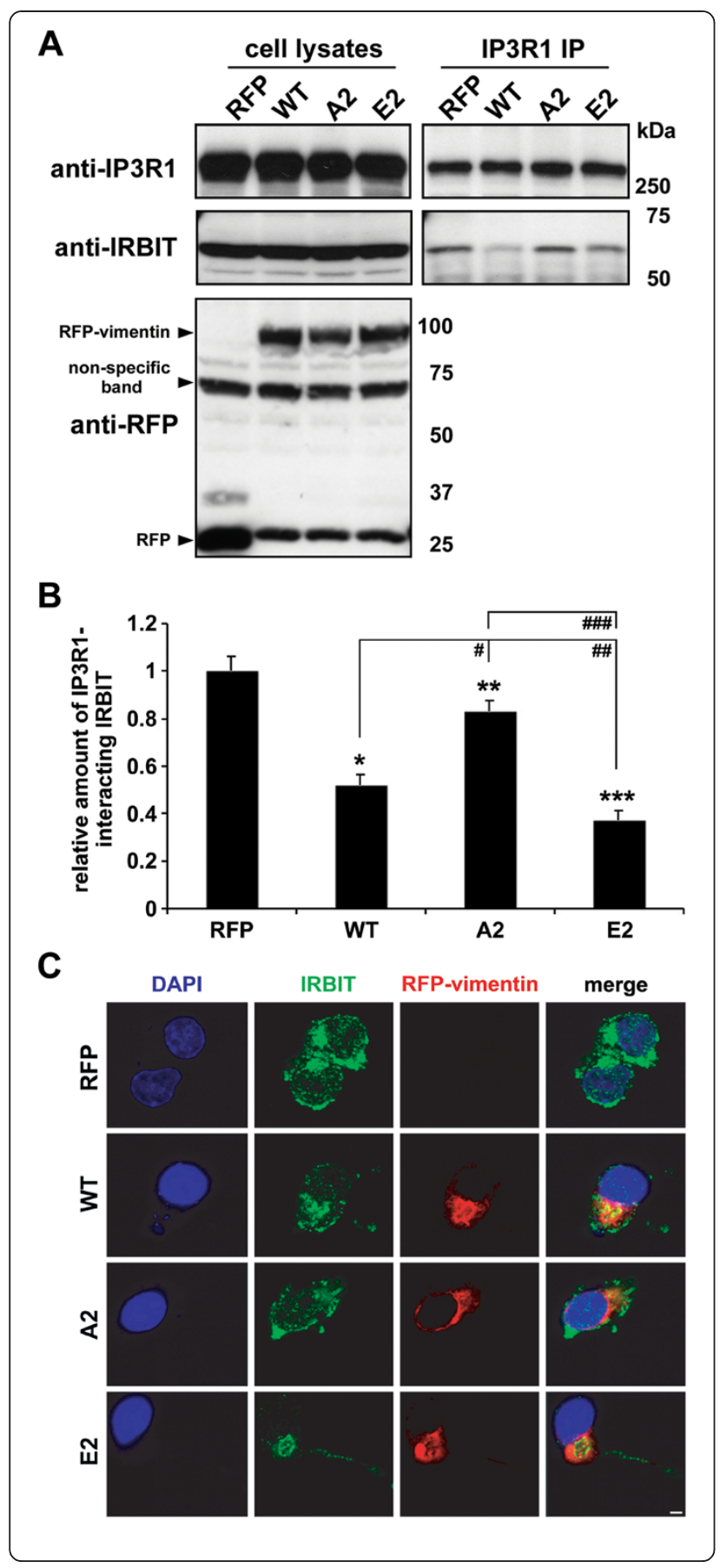

the polyQ toxicity (Figure 2D). We next analyzed the anti-aggregation effect of WT and phospho-mutants of vimentin in 150Q Neuro2a cells. Ser71 and Ser38 were substituted with phosphomimetic Glu (E2 mutant) or non-phosphorylated Ala (A2 mutant) amino acid residues. Over-expression of any of the RFP-vimentin form increased inclusion formation in 150Q Neuro2a cells. The E2 and A2 mutants had significantly stronger and weaker effect, respectively, as compared to the WT vimentin.
Figure $\mathbf{3}$ Vimentin influences the IRBIT-IP3R1 interaction by sequestering IRBIT in perinuclear inclusions. A. HeLa cells were transfected with RFP or tested forms of RFP-vimentin. 24 hrs later, cells were lysed and immunopreciptitation was performed using 10A6 anti-IP3R1 antibody. For immunoblotting, KM1112 anti-IP3R1, anti-IRBIT and anti-RFP antibodies were used. B. Quantification of IRBIT-IP3R1 interaction. The densities of immunoprecipitated IRBIT were normalized to the densities of corresponding immunoprecipitated IP3R1. ${ }^{*} p=0.0002,{ }^{* *} p=0.009,{ }^{* * *} p=0.0001$, ${ }^{\#} p=0.0017,{ }^{\# \#} p=0.029, " \# p=0.0009$. Bars represent relative mean values \pm s.d. from three independent experiments, with levels of IRBIT-IP3R1 interaction under control conditions normalized to a value of 1. C. Representative confocal images show distribution of immobile fraction of IRBIT in presence of RFP or tested RFP-vimentin forms. Neuro2a cells were transfected with RFP or RFP-vimentins, 24 hrs later permeabilized with saponin and immunostained for endogenous IRBIT (green). Fluorescence of RFP (red) and DAPI (blue) are also shown. Note that RFP was washed out from cells during saponin permeabilization. Scale bar, $5 \mu \mathrm{m}$.

Importantly, the effect of Y-27632 was abolished in cells expressing vimentin mutants (Figure 2E). WT and E2 mutants significantly increased the number of dead cells removed from the wells during the preparation of the samples for ArrayScan analysis while A2 vimentin did not have significant effect as compared to the control cells transfected with RFP (Figure 2F). These results confirmed that the effect of ROCK inhibitor Y-27632 on the mutant Htt aggregation and cytotoxicity is mediated by the phosphorylation status of vimentin and partly depends on the levels of this protein.

\section{Vimentin sequesters IRBIT and decreases its interaction with IP3R1}

Next, we aimed to identify the mechanism, by which vimentin levels and phosphorylation modifies accumulation and aggregation of pathogenic Htt. Our hypothesis on vimentin affecting polyQ aggregation in cooperation with IP3R1 was based on several studies. Firstly, the phosphorylation dynamics plays an important role in vimentin network reorganization and it changes vimentin affinity to its interacting partners, mostly regulatory proteins, and their spatial distribution [42]. Secondly, IP3R1 is directly involved in mutant Htt inclusion formation [27]. Thirdly, there has been reported a crosstalk between IP3R1 activity and intermediate filaments [43]. It has also been suggested that IP3Rs may be involved in the mechanism underlying the potentiating action of the Y-27632 in neurite outgrowth [44], which includes modifications of vimentin dynamics [45].

As inhibiting IP3R1 activity reduced polyQ Htt accumulation and aggregation [27], it was feasible to assume that stimulation of IP3R1 can contribute to polyQ aggregation. While exploring this hypothesis, we focused on one of the IP3R regulatory proteins, IRBIT [46]. IRBIT 
binds to IP3R1 and prevents its activation by IP3 [23]. HeLa cells were transfected with RFP or tested forms of RFP-vimentin and 24 hrs later, immunoprecipitation using anti-IP3R1 antibody was performed. We found that over-expression of RFP-vimentin suppressed the IRBIT-IP3R1 interaction by $47 \%$ (Figure 3A, B). As the phosphorylation status of vimentin turned out important for the extent of the polyQ aggregation modification (Figure 2), we also wondered how the vimentin phosphomutants A2 and E2 would influence the IRBIT-IP3R1 interaction. As expected, the A2 mutant reduced this interaction only by $18 \%$ while the E2 form of vimentin suppressed the IRBIT binding to IP3R1 by $63 \%$ as compared to the control (Figure 3A, B). These findings suggested that both the levels and phosphorylation status of vimentin are determining factors in suppressing the IRBIT-IP3R1 interaction and therefore influencing the activity of IP3R1.

To support our observations, we investigated the localization of the membrane-bound fraction of IRBIT in the presence of vimentin. We transfected Neuro2a cells with RFP or with tested RFP-vimentin forms. The cells were then permeabilized with saponin to remove the soluble cytosolic proteins, and subjected to confocal microscopy with immunostained IRBIT. While RFP, as a soluble protein not interacting with cytoskeleton or membranes, was not detected in the samples, RFP-vimentin was present and displayed different localization patterns depending on the amino acids at positions 71 and 38. WT and particularly E2 vimentin formed perinuclear cage-like structures, while the A2 mutant was dispersed with mostly filamentous-like distribution (Figure 3C). Importantly, IRBIT appeared trapped inside the structures formed by WT and E2 RFP-vimentins with almost exclusive localization of IRBIT within these inclusions in the E2-transfected cells. The A2 mutant, on the other hand, did not affect the IRBIT distribution so markedly as compared to the control RFP-transfected cells (Figure 3C). These observations are in agreement with the data obtained by IRBITIP3R1 co-immunoprecipitation (Figure 3A, B).

\section{Modification of IRBIT sequestration by ROCK and UPS inhibition}

The next question was whether the distribution of tested RFP-vimentins and IRBIT could be modified upon ROCK or UPS inhibition by Y-27632 or MG132 treatment, respectively. In the untreated cells, E2 vimentin accumulated in perinuclear inclusions and colocalized with IRBIT. Diffuse cytoplasmic staining of IRBIT was markedly reduced as compared to control cells indicating that IRBIT was recruited by E2 mutant to the aggresome-like inclusions. The A2 mutant exerted filamentous-like distribution with most of IRBIT remaining diffuse. The distribution of WT vimentin appeared as an intermediate pattern between the mutants (Figure 4A). When cells were treated with Y-27632, the WT form gained a filamentous distribution

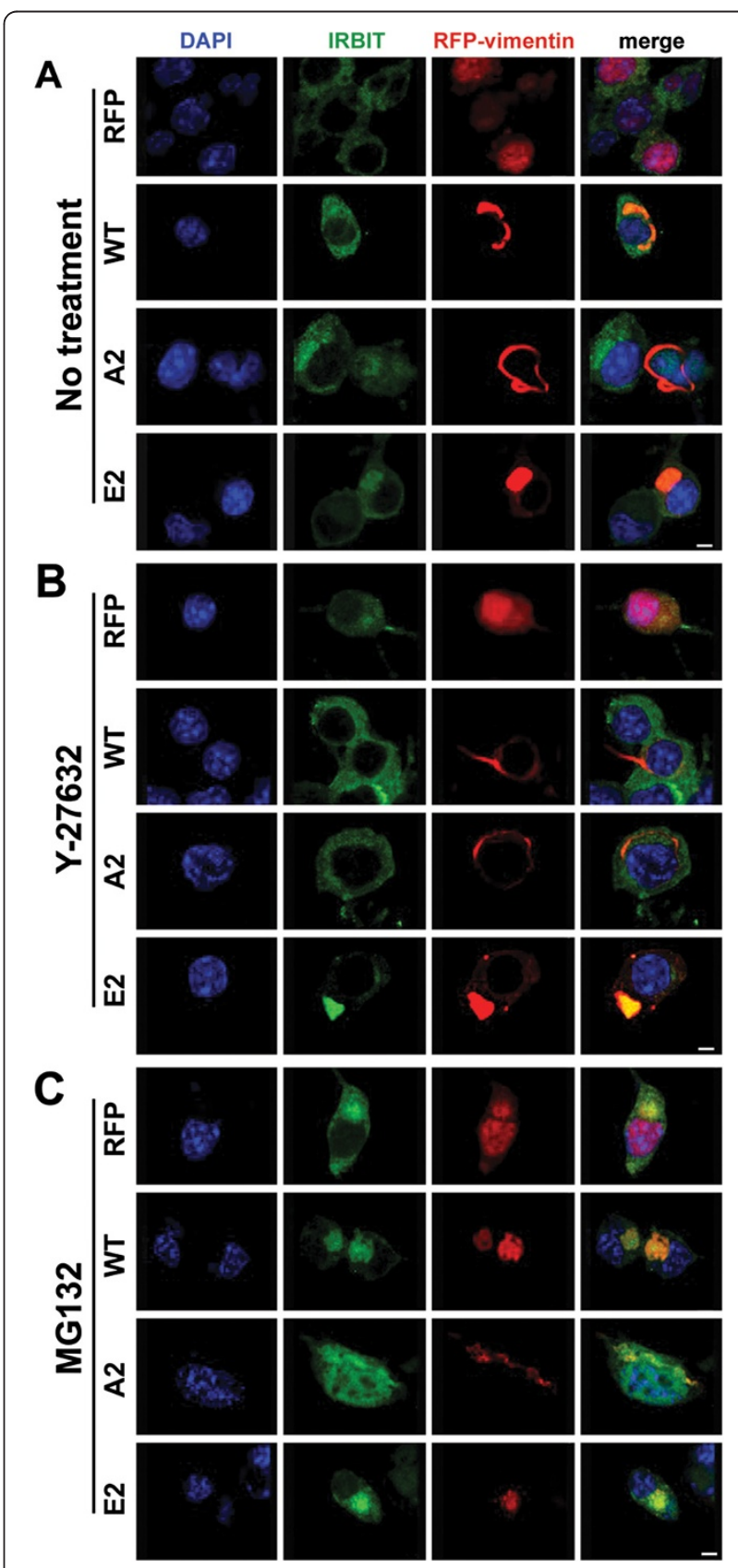

Figure 4 Vimentin phosphorylation affects IRBIT subcellular distribution. Effect of ROCK and UPS inhibitors. Representative confocal images show subcellular distribution of IRBIT in presence of RFP or tested RFP-vimentin forms. Neuro2a cells were transfected and treated as indicated for 24 hrs. Cells were fixed, permeabilized and immunostained for enogenous IRBIT (green). A. Control untreated cells (DMSO, 1/1000). B. Cells treated with $20 \mu \mathrm{M}$ Y-27632. C. Cells treated with $5 \mu \mathrm{M}$ MG132. Fluorescence of RFP (red) and DAPI (blue) are also shown. Scale bar, 5 um. 
(similar to A2 mutant at this and at the control condition) and lost the colocalization with IRBIT seen in the non-treated cells (Figure 4B). The unphosphorylated A2 and phosphomimetic E2 mutants were resistant to Y-27632 treatment (target serine amino acids mutated) and retained their distributions with A2 being filamentous-like, and E2 forming perinuclear aggresome-like inclusions and trapping IRBIT. These results suggest that the subcellular distribution of IRBIT regulated by vimentin is under the control of ROCK through phosphorylation of Ser71 and Ser38.

UPS inhibition has been shown to induce the formation of aggresomes [20]. Upon treatment with MG132, we observed IRBIT accumulation in aggresome-like structures even in control cells transfected with RFP. UPS inhibition in cells expressing WT RFP-vimentin caused its complete relocation into perinuclear inclusions and IRBIT accumulation in this location was markedly enhanced as compared to control cells, resembling the effect of E2 RFP-vimentin (Figure 4C). In the E2transfected cells, this distribution of vimentin and IRBIT was observed under all conditions (Figure 4A-C). Unexpectedly, the A2 mutant appeared to be resistant to MG132 treatment, retained its filamentous structure and prevented the accumulation of IRBIT in the aggresomelike inclusions (Figure 4C). This observation suggested a novel role for vimentin as a component actively regulating aggresome formation or at least sequestering and immobilizing certain proteins within this structure. We hypothesize that when the vimentin cage is not fully formed, some of the proteins can escape from aggresomes and at least partially fulfill their function at the physiological subcellular locations.

Overall, our results suggest that IRBIT can be sequestered by vimentin to perinuclear aggresome-like structures. The extent of sequestration appears to depend not only on the levels but also on the phosphorylation status of vimentin. All the above-discussed observations on vimentin-IRBIT connection were obtained in the absence of mutant Htt to avoid possible influence of this pathogenic protein, as mutant $\mathrm{Htt}$ sensitizes IP3R1 to IP3 via direct binding to the C-terminal part of IP3R1 $[25,39]$ and augmenting aggresome formation [19].

\section{Effect of vimentin on mutant $\mathrm{Htt}$ aggregation is mediated by IRBIT}

To test the relevance of the vimentin-IRBIT pathway to HD, we examined whether IRBIT could be a mediator of the modifying effect of vimentin on mutant Htt aggregation. We over-expressed WT, A2 or E2 RFP-vimentin in 150Q Neuro2a cells with or without knocking-down IRBIT, induced the tNHtt-150Q-EGFP expression and analyzed the inclusion formation by ArrayScan. Knockdown of IRBIT increased the inclusion formation almost twice (1.95-fold) in the RFP-expressing cells. The effect of IRBIT knock-down was enhanced by all forms of vimentin with E2 mutant having the strongest effect followed by WT and the A2 mutant with 8.37-, 6.65- and 5.57-fold increase of inclusion formation, respectively (Figure 5A). Over-expression of IRBIT, in contrary, reduced the inclusion formation in 150Q Neuro2a cells by $35 \%$. The effect of high IRBIT levels was relatively reduced in the presence of WT and A2 vimentin, with $23 \%$ and $28 \%$ difference, respectively, between the single and double transfections. E2 vimentin, on the other hand, abolished the effect of IRBIT with no difference between the E2 and E2 + IRBIT conditions (Figure 5C). Examples of compound images generated by ArrayScan representing each experimental condition are shown in Figure $5 \mathrm{~B}$ and D.

These data suggest that over-expressed WT and particularly E2 vimentin sequester IRBIT and may impair its function, while the A2 mutant has a relatively mild inhibitory effect. This is in accordance with the results in Figures 3 and 4 showing phosphorylated vimentin trapping IRBIT in perinuclear structures more efficiently than the phospho-resistant, A2 form.

\section{Conclusions}

In the present study, we introduce vimentin as a modifier of mutant $\mathrm{Htt}$ aggregation. Vimentin over-expression increased and the knock-down reduced the mutant $\mathrm{Htt}$ aggregation in Neuro2a cells. ROCK inhibitor Y-27632 inhibited vimentin phosphorylation at Ser71 and Ser38 and reduced the promoting effect of vimentin on mutant Htt aggregation. We found that interaction of IRBIT with IP3R1 is affected by vimentin and that the extent of this effect is dependent on the amino acids at positions 71 and 38. Accordingly, vimentin sequestered IRBIT in cage-like structures resembling aggresomes with the phosphomimetic E2 vimentin mutant traping IRBIT almost exclusively in perinuclear inclusions. The unphosphorylated A2 mutant expression, on the other hand, did not result in cage formation and IRBIT sequestration even when UPS was inhibited. We showed the relevance of vimentin-IRBIT axis in polyQ aggregation regulation in $150 \mathrm{Q}$ Neuro2a cells, where reduced levels of IRBIT enhanced, and increased levels of IRBIT decreased mutant Htt inclusion formation. These effects were modified by vimentin levels and mutations at Ser71 and Ser38. Although it has been speculated that aggresomes fulfill a protective role in polyQ diseases pathomechanism [47], based on our study we hypothesize that this function might depend on the dynamics of aggresome formation. If normal cellular proteins are sequestered too fast to the aggresomes without a sufficient time period for the cell to replace them or adapt to this state, it may contribute to cell death. 


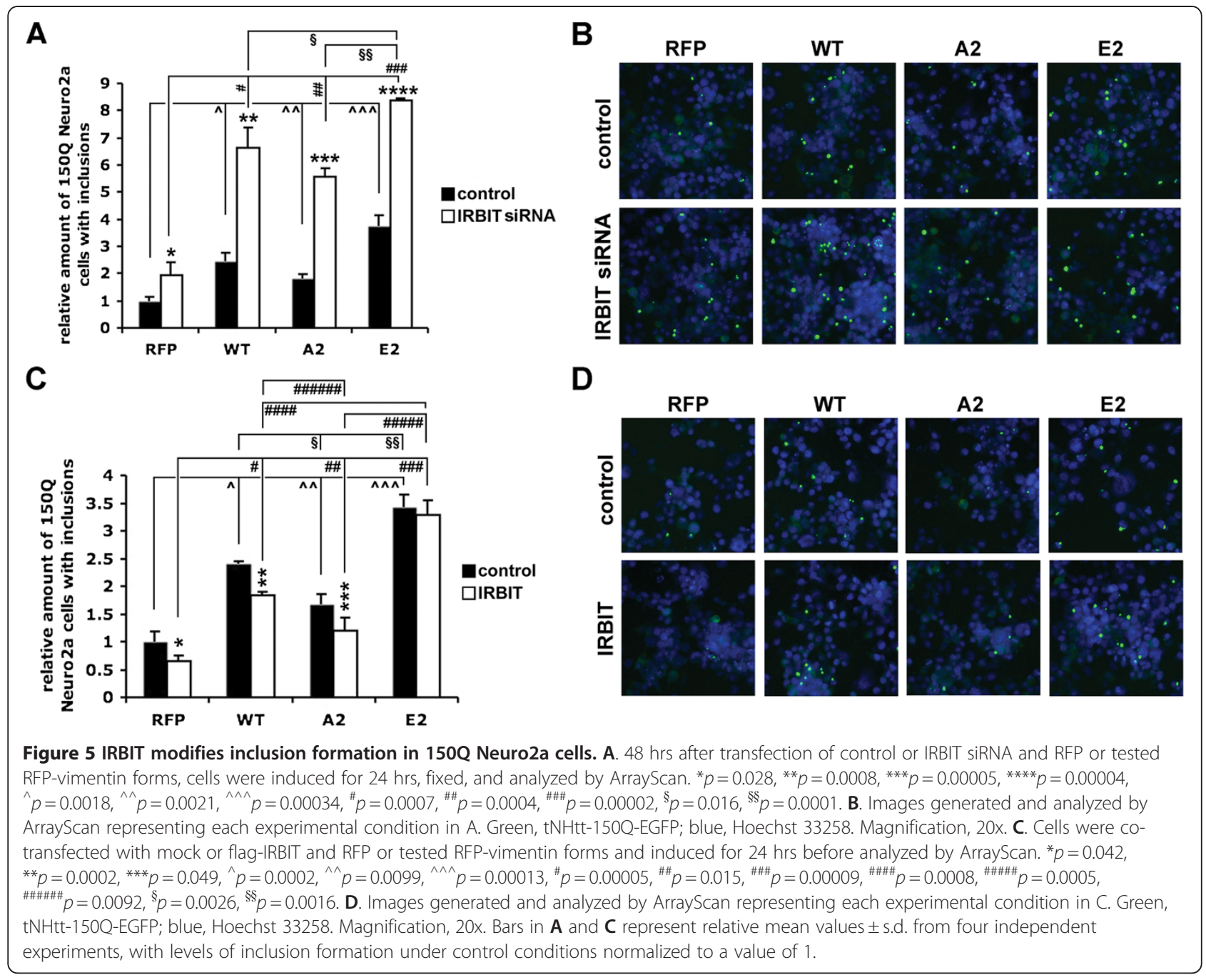

We would like to propose the following mechanistic model for the modifying effect of vimentin on polyQ protein accumulation and inclusion formation: vimentin and preferentially its phosphorylated form (at Ser71 and Ser38) promote polyQ aggregation by sequestering IRBIT in perinuclear inclusions and preventing its interaction with IP3R1 (Figure 6A). Increased activity of IP3R1 in polyQ diseases models has been previously reported to contribute to cytotoxicity of the pathogenic misfolded proteins $[25,26,28]$ and knock-down or chemical inhibition of IP3R1 reduced mutant Htt aggregation [27]. The therapeutic potential of ROCK inhibitors may thus be partly mediated by decreased vimentin phosphorylation leading to reduction of IP3R1 activity (Figure 6B). Importantly, activation of vimentin expression was shown in mature neurons affected by neurodegenerative or traumatic insults [15,16]. Reduction of vimentin levels and/or phosphorylation appears as a promising therapeutic strategy for $\mathrm{HD}$ and other polyQ diseases.

\section{Methods}

\section{Materials}

The ROCK inhibitor Y-27632 was from Sigma and MG132 (Z-Leu-Leu-Leu-aldehyde) from Wako Chemicals. Fluorescent nucleic acid stain Hoechst 33258 was from Molecular Probes. Mouse monoclonal antibody recognizing expanded polyQ tract, $1 \mathrm{C} 2$, and rat monoclonal anti- $\beta$-tubulin were obtained from Chemicon. Mouse monoclonal anti-vimentin antibody antibody was from Sigma. Rat monoclonal anti-phospho-vimentin (Ser71) and (Ser38) antibodies, and mouse monoclonal anti-GFP and anti-RFP antibodies were purchased from MBL. Mouse monoclonal anti-IP3R1 antibody KM1112, rat anti-IP3R1 antibody 10A6 and rabbit anti-IRBIT were generated as reported previously $[22,48,49]$.

\section{Plasmids}

Plasmids encoding the truncated N-terminal of human huntingtin (tNHtt) with 16,60 , and 150 glutamine repeats were introduced in pEGFP-N1 vector as previously 

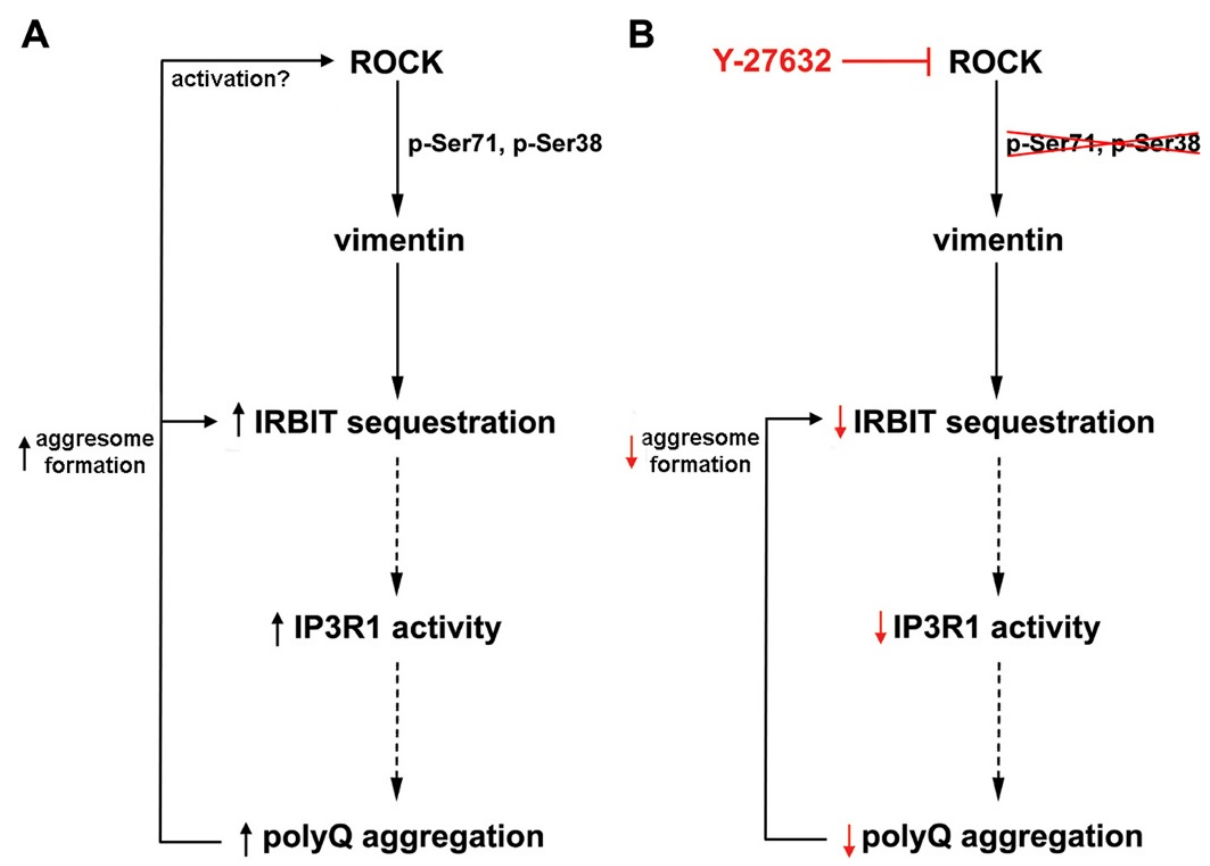

Figure 6 Schematic representation of the proposed mechanism of the vimentin effect on mutant Htt aggregation. A. In HD, mutant Htt and dopamine stimulation may activate ROCK, which in turn phosphorylates vimentin at Ser71 and Ser38 leading to IRBIT sequestration, reduced interaction of IRBIT with IP3R1, and activation of IP3R1. Mutant Htt expression and aggregation may increase aggresome formation enhancing IRBIT sequestration. B. Blocking vimentin phosphorylation by ROCK inhibitors may lead to reduced IRBIT sequestration by vimentin and consequently to decreased accumulation and aggregation of the pathogenic polyQ protein.

described [50]. To prepare pcDNA3.1-tNHtt-polyQ-EGFP with $60 \mathrm{Q}$ and $150 \mathrm{Q}$ for transient transfection, tNHttpolyQ-EGFP fragment was cut from pIND tNHtt-polyQEGFP [51] with HindIII-XbaI digestion, and the resulting fragment was inserted into pcDNA3.1-v5/His plasmid. The monomeric red fluorescence protein (RFP) plasmid preparation has previously been described [52]. Construction of N-terminally Flag-tagged IRBIT was described previously [23].

Mouse vimentin was amplified from mouse cDNA library using 5'-TCCCGAATTCAAGCTTCCACCATGT CTACCAGGTCTGTGTCC-3' as forward and 5'-AAA CACCGGATCCGGTTCAAGGTCATCGTGATGCTG-3' as reverse primer. The amplified vimentin $\mathrm{CDNA}$ fragment was inserted into HindIII/BamHI site of the pmRFP-C1 plasmid and named RFP-vimentin.

The mutations in RFP-vimentin were introduced using QuikChange $^{\circledR}$ Site-Directed Mutagenesis Kit (Stratagene). To generate phospho-mimetic (E2) and unphosphorylated (A2) mutants, following primers were used to exchange Ser71 and Ser38 to Glu or Ala: Ser71Glu: forward: 5`-GTGCGCCTGCGGGAAAGCGTGCCGGGCTG-3` and reverse, $5{ }^{`}$-CAGCCCGGCACGCTTTCCCGCAGGC GCAC-3` Ser38Glu: forward, 5` CACGTCCACACGCA CCTACGAACTGGGCAGCGCAC-3’ and reverse, 5'- GT GCGCTGCCCAGTTCGTAGGTGCGTGTGGACGTG-3; Ser71Ala: forward, 5'-GTGCGCCTGCG GGCTAGCGT
GCCGGGCTG-3`and reverse, $5{ }^{`}$-CAGCCCGGCACG CTAGCCCGCAGGCGCAC-3`. Ser38Ala: forward, 5` CACGTCCACACGCACCTACGCTCTGGGCAGCGC AC-3` and reverse, 5` - GTGCGCTGCCCAGAGCGTAG GTGCGTGTGGACGTG-3`.

Cell culture, transient transfection and treatments

Mouse neuroblastoma (Neuro2a) and human cervical carcinoma cells (HeLa) cells were maintained in Dulbecco's modified Eagle's medium (Sigma) supplemented with 10\% heat-inactivated fetal bovine serum (Sigma), $100 \mathrm{U} / \mathrm{ml}$ penicillin and $100 \mu \mathrm{g} / \mathrm{ml}$ streptomycin (Invitrogen) at $37^{\circ} \mathrm{C}$ in an humidified atmosphere containing $5 \% \mathrm{CO}_{2}$. Establishment of stable Neuro2a cell lines with the ecdysoneinducible mammalian expression system (Invitrogen), that express tNHtt-EGFP-16Q (16Q Neuro2a cells), tNHtt60Q-EGFP (60Q Neuro2a cells) and tNHtt-EGFP-150Q (150Q Neuro2a cells) has been described earlier [50,51]. Neuro2a cells were differentiated with $5 \mathrm{mM}$ dbcAMP $\left(N^{6}, 2^{\prime}\right.$-O-dibutyryladenosine-3',5'-cyclic monophosphate sodium salt) and induced to express tNHtt-polyQ-EGFP with $2 \mu \mathrm{M}$ ponasterone A (Invitrogen).

RFP-vimentin was transfected into Neuro2a/FRT cells [53]. The stably transfected cells resistant to treatment with $400 \mu \mathrm{g} / \mathrm{ml} \mathrm{G} 418$ (Calbiochem), were sub-cloned twice.

All transient transfections were performed when the cells reached $70-80 \%$ confluence with Lipofectamine 
2000 (Invitrogen) or Trans-IT (Mirus) according to the manufacturer's instruction.

\section{RNA interference}

The non-silencing control, vimentin and IP3R1 shRNAs were obtained from Open Biosystems. Plasmids were transfected into cells using Lipofectamine 2000. Neuro2a cells were induced $48 \mathrm{hrs}$ later. Stealth siRNA specific for IRBIT and scrambled control were obtained from Invitrogen. $20 \mu \mathrm{M}$ siRNA stock solutions were used for transfection to Neuro2a cells by Lipofectamine 2000 and after $48 \mathrm{hrs}$, cells were transfected again with RFP or RFPvimentin. Cells were used for experiments 24 hrs later.

\section{ArrayScan quantification}

For the quantification of the inclusions, cells were grown in 24-well plates, fixed in $4 \%$ paraformaldehyde, washed and incubated with Hoechst 33258 at 1/1000 dilution in PBS. Cells were analyzed by ArrayScan ${ }^{\circledR} \mathrm{V}^{\mathrm{TI}}$ High Content Screening (HCS) Reader (Cellomics) using Target Activation BioApplication (TABA) as described earlier [37]. TABA analyzes images acquired by a HCS Reader and provides measurements of the intracellular fluorescence intensity and localization on a cell-by-cell basis. In each well, at least 10,000 cells were counted and quantified for the presence of the inclusions. Scanning was performed with triplicate or quadruplicate in each experimental condition.

\section{Cell death assay}

For quantification of cell viability, $5 \mu \mathrm{g} / \mathrm{ml}$ each of Hoechst 33342 and PI were added to differentiated and induced Neuro2a cells. After $10 \mathrm{~min}$ at $37^{\circ} \mathrm{C}$, the PIpositive cells were quantified with ArrayScan.

\section{HeLa cells lysis and immunoprecipitation experiments}

Twenty four hours after transfection, HeLa cells were lysed in buffer containing $50 \mathrm{mM}$ Hepes ( $\mathrm{pH}$ 7.5), $150 \mathrm{mM} \mathrm{NaCl}, 2 \mathrm{mM}$ EDTA, Complete protease inhibitor cocktail (Roche) and 0.5\% NP40 (Sigma) for $30 \mathrm{~min}$ on ice and briefly sonicated. Cell lysates were centrifuged at $10,000 \mathrm{~g}$ for $30 \mathrm{~min}$ at $4^{\circ} \mathrm{C}$. Supernatants were rotated for 2 hrs at $4^{\circ} \mathrm{C}$ with IP3R1 antibody. Immuno-bound complexes were isolated by incubation with $20 \mu \mathrm{l}$ of protein G-Sepharose 4B beads (50\% slurry) (Amersham) for 2 hrs at $4^{\circ} \mathrm{C}$. Precipitated proteins were eluted with SDS-PAGE sample buffer and analyzed by western blotting with appropriate antibodies.

\section{Western blotting}

Cells were washed twice with ice-cold PBS, scraped, and resuspended in lysis buffer containing $0.5 \%$ Triton $\mathrm{X}-100$ in PBS (pH 7.4), $0.5 \mathrm{mM}$ phenylmethylsulfonyl fluoride and Complete protease inhibitor cocktail. After incubating on ice for $30 \mathrm{~min}$, lysates were briefly sonicated. Protein concentrations were determined according to the method of Bradford using Bio-Rad protein assay reagent (Bio-Rad) and the Western blot procedure was performed as described previously [37]. Images were quantified using Multi Gauge software (Fujifilm).

\section{Confocal microscopy}

Neuro2a cells were grown, transfected and treated in 4well chamber slides. Cells were processed according to two protocols. Firstly, permeabilization and fixation protocol was used to wash out cytosolic proteins not bound to membranes or cytoskeleton. Cells were washed with PBS followed incubation in ice-cold pre-extraction buffer containing $80 \mathrm{mM}$ PIPES (pH 7.2), $1 \mathrm{mM} \mathrm{MgCl}$, $1 \mathrm{mM}$ EGTA, 4\% PEG 6000 and $0.1 \%$ saponin on ice for $10 \mathrm{~min}$. Samples were rinsed with PBS and fixed with $4 \%$ formaldehyde in PBS for $15 \mathrm{~min}$ at room temperature. Secondly, standard procedure was used with cell fixation in $4 \%$ paraformaldehyde/PBS and permeabilization with $0.1 \%$ Triton X-100/PBS. Samples were incubated with anti-IRBIT antibody for $1 \mathrm{hr}$ at room temperature, washed, incubated for $1 \mathrm{hr}$ with Alexa Fluor 488 anti-rabbit secondary antibody (Invitrogen) and mounted with Vectashield mounting medium containing DAPI. Inducible tNHtt-polyQ-EGFP Neuro2a transfected with RFP or RFP-vimentin and Neuro2a cells stably expressing RFP-vimentin transfected with tNHtt16Q-EGFP or tNHtt-60Q-EGFP were fixed using $4 \%$ paraformaldehyde/PBS, and mounted with Vectashield mounting medium containing DAPI. Images were generated using confocal microscope (Leica).

\section{Statistical analysis}

Unpaired student's $t$-test for comparison between two samples was used. One-way ANOVA Fisher's test followed by Tukey's HSD test or two-way ANOVA test with pair-wise contrast was performed. The data was generated with XLSTAT software. We considered the difference between comparisons to be significant when $p<0.05$ for all statistical analyses.

\section{Additional file}

Additional file 1: Figure S1. Confocal images show distribution of pathogenic 150Q tNHtt (green) and RFP-vimentin (red) in inducible tNHtt-150Q-EGFP Neuro2a cells. Note the cages formed by vimentin around the $150 \mathrm{Q} \mathrm{tNH}$ tt aggregates. Nuclei were stained with DAPI (blue). scale bar, $10 \mu \mathrm{m}$

\section{Abbreviations}

HD: Huntington's Disease; Htt: Huntingtin; polyQ: Polyglutamine; IP3: Inositol 1,4,5-trisphosphate; IP3R1: IP3 receptor type 1; IRBIT: IP3R1-interacting protein released with IP3; UPS: Ubiquitin proteasome system; IF: Intermediate filament; WT: Wild-type; D2R: Dopamine D2 receptor; ROCK: Rho-associated kinase; PRK-2: Protein kinase C-related protein kinase-2; tNHtt: Truncated 
$\mathrm{N}$-terminal of human Htt; EGFP: Enhanced green fluorescent protein; RFP: Red fluorescence protein.

\section{Competing interests}

The authors declare that they have no competing interests.

\section{Authors' contributions}

$\mathrm{POB}$ and $\mathrm{RH}$ raised the hypothesis and designed the experiments. Experimental work was performed by POB, RH, AG and MK RFP-vimentin construct and the stable RFP-Vimentin Neuro2a cell line was prepared by GM The results were analyzed by POB, RH, AG, KM and NN The manuscript was written by POB, RH and NN. All authors discussed the results and commented on the manuscript.

\section{Acknowledgements}

This work was partly supported by a Grant from Japan Society for the Promotion of Science (JSPS). P.O.B. and R.H were JSPS postdoctoral fellows. This work was supported by Grant-in-Aid from the Ministry of Education, Culture, Sports, Science, and Technology of Japan for N.N. (22110004 and 22240037), by Core Research for Evolutional Science and Technology from Japan Science and Technology Agency for N.N., and by a Grant in-Aid for the Research on Measures for Ataxic Diseases from the Ministry of Health, Welfare and Labor for N.N.

\section{Author details}

'Laboratory for Structural Neuropathology, Brain Science Institute, RIKEN, 2-1 Hirosawa, Wako-shi, Saitama 351-0198, Japan. ${ }^{2}$ Laboratory for Developmental Neurobiology, Brain Science Institute, RIKEN, 2-1 Hirosawa, Wako-shi, Saitama 351-0198, Japan. ${ }^{3}$ Calcium Oscillation Project, ICORP-SORST, Japan Science and Technology Agency (JST), 4-1-8 Honcho, Kawaguchi, Saitama 332-0012, Japan. ${ }^{4}$ Present address: Neuro-Oncology Branch, National Cancer Institute, National Institute of Neurological Disorders and Stroke, National Institutes of Health, 37 Convent Drive, Bethesda, MD 20892, USA. ${ }^{5}$ Present address: Institute of Biochemistry, Nutrition and Health Protection, Department of Biochemistry and Microbiology, Faculty of Chemical and Food Technology, Slovak University of Technology, Radlinskeho 9, 81237 Bratislava, Slovakia.

Received: 16 May 2012 Accepted: 6 August 2012 Published: 28 August 2012

\section{References}

1. Myers RH: Huntington's disease genetics. NeuroRx 2004, 1:255-262.

2. Orr HT, Zoghbi HY: Trinucleotide repeat disorders. Annu Rev Neurosci 2007, 30:575-621.

3. The Huntington's Disease Collaborative Research Group: A novel gene containing a trinucleotide repeat that is expanded and unstable on Huntington's disease chromosomes. Cell 1993, 72:971-983.

4. Difiglia M, Sapp E, Chase KO, Davies SW, Bates GP, Vonsattel JP, Aronin N: Aggregation of huntingtin in neuronal intranuclear inclusions and dystrophic neurites in brain. Science 1997, 277:1990-1993.

5. Saudou F, Finkbeiner S, Devys D, Greenberg ME: Huntingtin acts in the nucleus to induce apoptosis but death does not correlate with the formation of intranuclear inclusions. Cell 1998, 95:55-66.

6. Landles C, Bates GP: Huntingtin and the molecular pathogenesis of Huntington's disease, Fourth in molecular medicine review series. EMBO Rep 2004, 5:958-963.

7. Bauer $\mathrm{PO}$, Nukina $\mathrm{N}$ : The pathogenic mechanisms of polyglutamine diseases and current therapeutic strategies. J Neurochem 2009, 110:1737-1765.

8. Oliveira JMA, Jekabsons MB, Chen S, Lin A, Rego CA, Goncalves J, Ellerby $L M$, Nicholls DJ: Mitochondrial dysfunction in Huntington's disease: the bioenergetics of isolated and in situ mitochondria from transgenic mice. J Neurochem 2007, 101:241-249.

9. Oliveira JMA: Mitochondrial bioenergetics and dynamics in Huntington's disease: tripartite synapses and selective striatal degeneration. $J$ Bioenerg Biomembr 2010, 42:227-234.

10. Franke WW, Schmid E, Winter S, Osborn M, Weber K: Widespread occurrence of intermediate-sized filaments of the vimentin-type in cultured cells from diverse vertebrates. Exp Cell Res 1979, 123:25-46.

11. Paulin D, Jakob H, Jacob F, Weber K, Osborn M: In vitro differentiation of mouse teratocarcinoma cells monitored by intermediate filament expression. Differentiation 1982, 22:90-99.
12. Bignami A, Raju T, Dahl D: Localization of vimentin, the nonspecific intermediate filament protein, in embryonal glia and in early differentiating neurons, In vivo and in vitro immunofluorescence study of the rat embryo with vimentin and neurofilament antisera. Dev Biol 1982, 91:286-295.

13. Franke WW, Schmid E, Osborn M, Weber K: Intermediate-sized filaments of human endothelial cells. J Cell Biol 1979, 81:570-580.

14. Izmiryan A, Franco CA, Paulin D, Li Z, Xue Z: Synemin isoforms during mouse development: multiplicity of partners in vascular and neuronal systems. Exp Cell Res 2009, 315:769-783.

15. Yen SH, Gaskin F, Fu SM: Neurofibrillary tangles in senile dementia of the Alzheimer type share an antigenic determinant with intermediate filaments of the vimentin class. Am J Pathol 1983, 113:373-381.

16. Levin EC, Acharya NK, Sedeyn JC, Venkataraman V, D'Andrea MR, Wang HY, Nagele RG: Neuronal expression of vimentin in the Alzheimer's disease brain may be part of a generalized dendritic damage-response mechanism. Brain Res 2009, 1298:194-207.

17. Wigley WC, Fabunmi RP, Lee MG, Marino CR, Muallem S, DeMartino GN, Thomas PJ: Dynamic association of proteasomal machinery with the centrosome. J Cell Biol 1999, 145:481-490.

18. Ward CL, Omura S, Kopito RR: Degradation of CFTR by the ubiquitinproteasome pathway. Cell 1995, 83:121-127.

19. Johnston JA, Ward CL, Kopito RR: Aggresomes: a cellular response to misfolded proteins. J Cell Biol 1998, 143:1883-1898.

20. Waelter $S$, Boeddrich A, Lurz R, Scherzinger E, Lueder G, Lehrach $H$, Wanker EE: Accumulation of mutant huntingtin fragments in aggresome-like inclusion bodies as a result of insufficient protein degradation. Mol Biol Cell 2001, 12:1393-1407.

21. Shimohata T, Sato A, Burke JR, Strittmatter WJ, Tsuji S, Onodera O: Expanded polyglutamine stretches form an 'aggresome'. Neurosci Lett 2002, 323:215-218.

22. Ando H, Mizutani A, Matsu-ura T, Mikoshiba K: IRBIT, a novel inositol 1,4,5-trisphosphate (IP3) receptor-binding protein, is released from the IP3 receptor upon IP3 binding to the receptor. J Biol Chem 2003, 278:10602-10612.

23. Ando H, Mizutani A, Kiefer H, Tsuzurugi D, Michikawa T, Mikoshiba K: IRBIT suppresses IP3 receptor activity by competing with IP3 for the common binding site on the IP3 receptor. Mol Cell 2006, 22:795-806.

24. Devogelaere B, Beullens M, Sammels E, Derua R, Waelkens E, Van Lint J, Parys JB, Missiaen L, Bollen M, De Smedt H: Protein phosphatase-1 is a novel regulator of the interaction between IRBIT and the inositol 1,4,5trisphosphate receptor. Biochem J 2007, 407:303-311.

25. Tang TS, Tu H, Chan EY, Maximov A, Wang Z, Wellington CL, Hayden MR, Bezprozvanny l: Huntingtin and huntingtin-associated protein 1 influence neuronal calcium signaling mediated by inositol- $(1,4,5)$ triphosphate receptor type 1. Neuron 2003, 39:227-239.

26. Bezprozvanny I: Inositol 1,4,5-tripshosphate receptor, calcium signaling and Huntington's disease. Subcell Biochem 2007, 45:323-335.

27. Bauer PO, Hudec R, Ozaki S, Okuno M, Ebisui E, Mikoshiba K, Nukina N: Genetic ablation and chemical inhibition of IP3R1 reduce mutant huntingtin aggregation. Biochem Biophys Res Commun 2011, 416:13-17.

28. Tang TS, Slow E, Lupu V, Stavrovskaya IG, Sugimori M, Llinás R, Kristal BS, Hayden MR, Bezprozvanny I: Disturbed Ca2+ signaling and apoptosis of medium spiny neurons in Huntington's disease. Proc Natl Acad Sci USA 2005, 102:2602-2607.

29. Goto H, Kosako H, Tanabe K, Yanagida M, Sakurai M, Amano M, Kaibuchi K, Inagaki M: Phosphorylation of vimentin by Rho-associated kinase at a unique amino-terminal site that is specifically phosphorylated during cytokinesis. J Biol Chem 1998, 273:11728-11736.

30. Nakamura Y, Hashimoto R, Amano M, Nagata K, Matsumoto N, Goto H, Fukusho E, Mori H, Kashiwagi Y, Kudo T, Inagaki M, Takeda M: Localized phosphorylation of vimentin by rho-kinase in neuroblastoma N2a cells. Genes Cells 2000, 5:823-837.

31. Ishizaki T, Maekawa M, Fujisawa K, Okawa K, Iwamatsu A, Fujita A, Watanabe N, Saito Y, Kakizuka A, Morii N, Narumiya S: The small GTP-binding protein Rho binds to and activates a $160 \mathrm{kDa}$ Ser/Thr protein kinase homologous to myotonic dystrophy kinase. EMBO J 1996, 15:1885-1893.

32. Matsui T, Amano M, Yamamoto T, Chihara K, Nakafuku M, Ito M, Nakano T, Okawa K, Iwamatsu A, Kaibuchi K: Rho-associated kinase, a novel serine/ threonine kinase, as a putative target for small GTP binding protein Rho. EMBO J 1996, 15:2208-2216. 
33. Riento K, Ridley AJ: Rocks: multifunctional kinases in cell behavior. Nat Rev Mol Cell Biol 2003, 4:446-456.

34. Pollitt SK, Pallos J, Shao J, Desai UA, Ma AA, Thompson LM, Marsh JL, Diamond MI: A rapid cellular FRET assay of polyglutamine aggregation identifies a novel inhibitor. Neuron 2003, 40:685-694.

35. Shao J, Welch WJ, Diamond MI: ROCK and PRK-2 mediate the inhibitory effect of Y-27632 on polyglutamine aggregation. FEBS Lett 2008, 582:1637-1642.

36. Shao J, Welch WJ, Diprospero NA, Diamond MI: Phosphorylation of profilin by ROCK1 regulates polyglutamine aggregation. Mol Cell Biol 2008, 28:5196-5208.

37. Bauer PO, Wong HK, Oyama F, Goswami A, Okuno M, Kino Y, Miyazaki H, Nukina N: Inhibition of Rho kinases enhances the degradation of mutant huntingtin. J Biol Chem 2009, 284:13153-13164.

38. Bauer $\mathrm{PO}$, Nukina $\mathrm{N}$ : Enhanced degradation of mutant huntingtin by rho kinase inhibition is mediated through activation of proteasome and macroautophagy. Autophagy 2009, 5:747-748.

39. Tang TS, Chen X, Liu J, Bezprozvanny I: Dopaminergic signaling and striatal neurodegeneration in Huntington's Disease. J Neurosci 2007, 27:7899-7910.

40. Deyts C, Galan-Rodriguez B, Martin E, Bouveyron N, Roze E, Charvin D, Caboche J, Bétuing S: Dopamine D2 receptor stimulation potentiates polyQ-Huntingtin-induced mouse striatal neuron dysfunctions via Rho/ ROCK-II activation. PLoS One 2009, 4:e8287.

41. Li M, Huang Y, Ma AA, Lin E, Diamond MI: Y-27632 improves rotarod performance and reduces huntingtin levels in R6/2 mice. Neurobiol Dis 2009, 3:413-420

42. Ivaska J, Pallari HM, Nevo J, Eriksson JE: Novel functions of vimentin in cell adhesion, migration, and signaling. Exp Cell Res 2007, 313:2050-2062.

43. Heimfarth L, Loureiro SO, Reis KP, de Lima BO, Zamboni F, Gandolfi T, Narvaes $R$, da Rocha JB, Pessoa-Pureur R: Cross-talk among intracellular signaling pathways mediates the diphenyl ditelluride actions on the hippocampal cytoskeleton of young rats. Chem Res Toxicol 2011, 24:1754-1764.

44. Minase T, Ishima T, Itoh K, Hashimoto K: Potentiation of nerve growth factor-induced neurite outgrowth by the ROCK inhibitor Y-27632: a possible role of IP3 receptors. Eur J Pharmacol 2010, 648:67-73.

45. Toth C, Shim SY, Wang J, Jiang Y, Neumayer G, Belzil C, Liu WQ, Martinez J, Zochodne D, Nguyen MD: Ndel1 promotes axon regeneration via intermediate filaments. PLOS One 2008, 3:e2014

46. Mikoshiba K: IP3 receptor/Ca ${ }^{2+}$ channel: from discovery to new signaling concepts. J Neurochem 2007, 102:1426-1446.

47. Taylor JP, Tanaka F, Robitschek J, Sandoval CM, Taye A, Markovic-Plese S, Fischbeck KH: Aggresomes protect cells by enhancing the degradation of toxic polyglutamine-containing protein. Hum Mol Genet 2003, 12:749-757.

48. Sugiyama T, Furuya A, Monkawa T, Yamamoto-Hino M, Satoh S, Ohmori K, Miyawaki A, Hanai N, Mikoshiba K, Hasegawa M: Monoclonal antibodies distinctively recognizing the subtypes of inositol 1,4,5-trisphosphate receptor: application to the studies on inflammatory cells. FEBS Lett 1994, 354:149-154.

49. Maeda N, Niinobe M, Mikoshiba K: A cerebellar Purkinje cell marker P400 protein is an inositol 1,4,5-trisphosphate (InsP3) receptor protein. Purification and characterization of InsP3 receptor complex. EMBO J 1990, 9:61-67.

50. Wang GH, Mitsui K, Kotliarova S, Yamashita A, Nagao Y, Tokuhiro S, Iwatsubo T, Kanazawa I, Nukina N: Caspase activation during apoptotic cell death induced by expanded polyglutamine in N2a cells. Neuroreport 1999, 10:2435-2438.

51. Zemskov EA, Jana NR, Kurosawa M, Miyazaki H, Sakamoto N, Nekooki M, Nukina N: Pro-apoptotic protein kinase $C$ delta is associated with intranuclear inclusions in a transgenic model of Huntington's disease. J Neurochem 2003, 87:395-406.

52. Machida Y, Okada T, Kurosawa M, Oyama F, Ozawa K, Nukina N: rAAVmediated shRNA ameliorated neuropathology in Huntington disease model mouse. Biochem Biophys Res Commun 2006, 343:190-197.

53. Matsumoto G, Wada K, Okuno M, Kurosawa M, Nukina N: Serine 403 phosphorylation of p62/SQSTM1 regulates selective autophagic clearance of ubiquitinated proteins. Mol Cell 2011, 44:279-289.

doi:10.1186/1750-1326-7-43

Cite this article as: Bauer et al:: ROCK-phosphorylated vimentin modifies mutant huntingtin aggregation via sequestration of IRBIT. Molecular Neurodegeneration 2012 7:43.

\section{Submit your next manuscript to BioMed Central and take full advantage of:}

- Convenient online submission

- Thorough peer review

- No space constraints or color figure charges

- Immediate publication on acceptance

- Inclusion in PubMed, CAS, Scopus and Google Scholar

- Research which is freely available for redistribution 